\title{
Ultranarrow-Band Synthetic Aperture Radar Imaging for Arbitrary Flight Trajectories
}

\author{
Ling Wang \\ College of Electronic and Information Engineering, \\ Nanjing University of Aeronautics and Astronautics, \\ Nanjing 210016, China \\ Email: wanglrpi@gmail.com
}

\author{
Birsen Yazıc1** \\ Department of Electrical, Computer and Systems Engineering, \\ Rensselaer Polytechnic Institute, \\ 110 8th Street, Troy, NY 12180 USA \\ Email: yazici@ecse.rpi.edu
}

\begin{abstract}
We present a novel image formation method for synthetic aperture radar (SAR) using ultra-narrowband continuous waveforms. Considering the high Doppler resolution nature of the ultra-narrowband continuous wave $(\mathrm{CW})$ signals, we refer to the SAR system using ultra-narrowband CW signals as Doppler Synthetic Aperture Radar (DSAR). We first correlate the translated version of the received signal with a scaled or frequency-shifted version of the transmitted signal over a finite time window, and then use microlocal analysis to reconstruct the scene by a filtered-backprojection (FBP) of the correlated signals. We show that the resolution of the image is directly related to the length of the support of the windowing function, the carrier-frequency of the transmitted waveform, and the sampling rate of the aperture. Unlike the previous approaches in the literature, our approach backprojects the correlated received signal onto iso-Doppler curves as opposed to iso-range curves, and takes advantage of the velocity, as well as the acceleration of the antennas in a certain direction to form a high resolution SAR image. Furthermore, it can accommodate arbitrary flight trajectories. We present numerical experiments to demonstrate the performance of the new image formation method.
\end{abstract}

\section{INTRODUCTION}

We consider a synthetic aperture radar imaging system where the transmitter transmits ultra-narrowband or singlefrequency continuous waveforms. Due to the high-Dopplerresolution nature of the transmitted waveforms, we refer to the system under consideration as Doppler synthetic aperture radar (DSAR).

As compared to the traditional SAR system that relies on high range resolution achieved by wideband transmission, DSAR requires a relatively simple and low-cost transmitter, and in some cases does not even need a dedicated transmitter. Existing electronic signals, such as radio and television signals and cell phone signals can be used as the illumination sources of DSAR. These waveforms are continuous, ultra-narrowband, and have high Doppler resolution.

The imaging of rotating objects using a single-frequency continuous wave $(\mathrm{CW})$ illumination has been investigated before in [1], [2]. This work was recently studied experimentally in [3]. The imaging of stationary objects using

This work was supported by the Air Force Office of Scientific Research (AFOSR) under the agreement FA9550-09-1-0013, and National Science Foundation (NSF) under Grant No. CCF-08030672.

** Corresponding author ultra-narrowband or narrowband $\mathrm{CW}$ with spatially distributed radars has been studied in [4], [5]. All of these studies use tomographic image formation methods that rely on trading the resolution achieved by bandwidth with the resolution achieved by spatial diversity. An alternative approach to mono-static synthetic aperture imaging using high-Dopplerresolution measurements was discussed in [6]. However, both the resolution analysis and the imaging method presented are limited to the straight flight path and flat topography. Furthermore, an approximate implementation of the imaging method based on Radon-Hough transform relying on detecting the lines associated with the scatterers has limited applicability in practice.

We present a novel filtered-backprojection (FBP) type image formation method for bistatic DSAR. The forward model is formed by correlating the translated version of the received signal with a scaled or frequency-shifted version of the transmitted signal over a finite time window. The high-frequency analysis of the resulting model shows that the correlated received signal is the projections of the scene onto the bistatic iso-Doppler curves. We use microlocal techniques to develop a FBP reconstruction of the scene. The analysis of the point spread function (PSF) of the imaging operator shows that the reconstructed images preserve the location and orientation of the singularities at the intersection of the bistatic iso-Doppler and iso-Doppler-rate curves. We show that the resolution of the image is directly related to the length of the support of the windowing function, the carrier-frequency of the transmitted waveform, and the sampling rate of the aperture. The method is applicable to arbitrary, but known flight trajectories and nonflat topography.

Our method has the following advantages: (1) Unlike the previous approaches in the literature, [1]-[5], our approach backpropagates the correlated received signal onto iso-Doppler curves as opposed to iso-range curves, and takes advantage of the velocity as well as the acceleration of the antennas in a certain direction to form a high resolution SAR image using ultra-narrowband waveforms; (2) it can be used under nonideal imaging scenarios such as arbitrary flight trajectories and non-flat topography; (3) it does not require antennas with high directivity; (4) it has the desirable property of preserving the visible edges of the scene in the reconstructed image; (5) it 
is an analytic reconstruction technique which can be made computationally efficient.

The organization of the paper is as follows: In Section II, we present the model for the received signal and develop the forward model for the bistatic DSAR. In Section III, we develop a filtered backprojection-type image formation method for bistatic DSAR and analyze the resolution of DSAR imaging. In Section IV, we conduct numerical simulations to demonstrate the performance of our DSAR imaging method. In Section $\mathrm{V}$, we conclude our discussion.

We use the following notational conventions throughout the paper. The bold Roman, bold italic and Roman lowercase letters are used to denote variables in $\mathbb{R}^{3}, \mathbb{R}^{2}$ and $\mathbb{R}$, respectively, i.e., $\mathbf{z}=(\boldsymbol{z}, z) \in \mathbb{R}^{3}$, with $\boldsymbol{z} \in \mathbb{R}^{2}$ and $z \in \mathbb{R}$. The calligraphic letters $(\mathcal{F}, \mathcal{K}$ etc. $)$ are used to denote operators.

\section{FORWARD MODEL FOR IMAGING}

For a pair of transmitter and receiver antennas located at $\mathbf{T}$ and $\mathbf{R}$ respectively, the received signal is modeled by [7]

$$
\begin{aligned}
f(t, \mathbf{R}, \mathbf{T})= & \int \frac{\mathrm{e}^{\mathrm{i} \omega\left(t-(|\mathbf{R}-\mathbf{z}|+|\mathbf{z}-\mathbf{T}|) / c_{0}\right)}}{(4 \pi)^{2}|\mathbf{R}-\mathbf{z}||\mathbf{z}-\mathbf{T}|} \omega^{2} \hat{p}(\omega) \\
& \times J_{\operatorname{tr}}(\omega, \mathbf{z}, \mathbf{T}) J_{\mathrm{rc}}(\omega, \mathbf{z}, \mathbf{R}) V(\mathbf{z}) d \omega d \mathbf{z},
\end{aligned}
$$

where $t$ denotes time, $\hat{p}$ denote the Fourier transform of transmitted waveform $p(t), c_{0}$ denotes the speed of light in free space, $V(\mathbf{z})$ is the reflectivity function, and $J_{t r}$ and $J_{r c}$ are the transmitter and receiver antenna beam pattern related terms, respectively.

For a narrow band waveform, we have

$$
p(t)=\mathrm{e}^{\mathrm{i} \omega_{0} t} \tilde{p}(t)
$$

where $\omega_{0}$ denotes the carrier frequency and $\tilde{p}(t)$ is the complex envelope of $p$, which is slow varying as a function of $t$ compared to $\mathrm{e}^{\mathrm{i} \omega_{0} t}$. Using (2) and under the assumption of broadband antenna, (1) becomes

$$
\begin{aligned}
& f(t, \mathbf{R}, \mathbf{T}) \approx \int \mathrm{e}^{\mathrm{i} \omega_{0}\left(t-(|\mathbf{R}-\mathbf{z}|+|\mathbf{z}-\mathbf{T}|) / c_{0}\right)} \\
& \quad \times \tilde{p}\left(t-(|\mathbf{R}-\mathbf{z}|+|\mathbf{z}-\mathbf{T}|) / c_{0}\right) A_{T R}\left(\omega_{0}, \mathbf{z}, \mathbf{R}, \mathbf{T}\right) V(\mathbf{z}) d \mathbf{z} .
\end{aligned}
$$

where $A_{T R}$ is given by

$$
A_{T R}=\frac{\omega_{0}^{2}}{(4 \pi)^{2}|\mathbf{R}-\mathbf{z}||\mathbf{z}-\mathbf{T}|} J_{\mathrm{tr}}\left(\omega_{0}, \mathbf{z}, \mathbf{T}\right) J_{\mathrm{rc}}\left(\omega_{0}, \mathbf{z}, \mathbf{R}\right)
$$

Let the earth's surface denoted by $\mathbf{z}=(\boldsymbol{z}, \boldsymbol{\psi}(\boldsymbol{z})) \in \mathbb{R}^{3}$, where $\boldsymbol{z} \in \mathbb{R}^{2}$ and $\boldsymbol{\psi}: \mathbb{R}^{2} \rightarrow \mathbb{R}$ is a known function for the ground topography. Furthermore, we assume that the scattering takes place in a thin region near the surface. Thus, the reflectivity function has the form

$$
V(\mathbf{z})=\rho(\boldsymbol{z}) \delta(z-\boldsymbol{\psi}(\boldsymbol{z}))
$$

For the rest of our discussion, we consider a bistatic SAR (BISAR) imaging system. Let $\gamma_{T}(t)$ and $\gamma_{R}(t)$ be the transmitter and receiver trajectories, respectively, and let $s(t)$ denote the received signal of the receiver. We then have $s(t)=f\left(t, \gamma_{R}(t), \gamma_{T}(t)\right)$. Using (3), the received signal for BISAR with transmitter transmitting an ultranarrow-band CW waveform becomes

$$
\begin{array}{r}
s(t)=\int \mathrm{e}^{\mathrm{i} \omega_{0}\left(t-R_{T R}(t, \boldsymbol{z}) / c_{0}\right)} \tilde{p}\left(t-R_{T R}(t, \boldsymbol{z}) / c_{0}\right) \\
\times A_{T R}\left(\omega_{0}, \mathbf{z}, \boldsymbol{\gamma}_{R}(t), \gamma_{T}(t)\right) \rho(\boldsymbol{z}) d \boldsymbol{z}
\end{array}
$$

where $R_{T R}(t, \boldsymbol{z})=\left|\gamma_{T}(t)-\mathbf{z}\right|+\left|\mathbf{z}-\gamma_{R}(t)\right|$ is the bistatic range, and $A_{T R}\left(\omega_{0}, \mathbf{z}, \gamma_{R}(t), \gamma_{T}(t)\right)$ is given by (4).

We define the correlation of the received signal given by (6) with a scaled or frequency-shifted version of the transmitted signal over a finite time window by

$$
d(\tau, \mu)=\int s(t+\tau) p^{*}(\mu t) \phi(t) d t
$$

for some $\tau \in \mathbb{R}$ and $\mu \in \mathbb{R}^{+}$, where $\phi(t)$ is a smooth windowing function centered at $t=0$ with finite support.

Substituting (6) and (2) into (7) and performing the Taylor series expansion at $t=0$ on $\gamma_{T}(t+\tau)$ and $\gamma_{R}(t+\tau)$, and rearranging some terms, we have

$$
\begin{aligned}
& d(\tau, \mu) \approx \int \mathrm{e}^{-\mathrm{i} \omega_{0} t\left[\mu-1+\left(\boldsymbol{\gamma}_{T}(\tau)-\mathbf{z} \cdot \dot{\gamma}_{T}(\tau)+\widehat{\boldsymbol{\gamma}_{R}(\tau)-} \mathbf{z} \cdot \dot{\gamma}_{R}(\tau)\right) / c_{0}\right]} \\
& \times \mathrm{e}^{\mathrm{i} \omega_{0}\left(\tau-R_{T R}(\tau, \boldsymbol{z}) / c_{0}\right)} \\
& \times \tilde{p}\left[\left(1-\left(\left(\gamma_{T} \widehat{(\tau)}-\mathbf{z} \cdot \dot{\gamma}_{T}(\tau)+\widehat{\gamma_{R}(\tau)}-\mathbf{z} \cdot \dot{\gamma}_{R}(\tau)\right) / c_{0}\right)\right) t\right. \\
& \left.+\tau-R_{T R}(\tau, \mathbf{z}) / c_{0}\right] \tilde{p}^{*}(\mu t) \\
& \times A_{T R}\left(\omega_{0}, \mathbf{z}, \gamma_{R}(t+\tau), \gamma_{T}(t+\tau)\right) \rho(\boldsymbol{z}) d \boldsymbol{z} \phi(t) d t .
\end{aligned}
$$

We write the forward model for bistatic DSAR as follows:

$$
\begin{aligned}
d(\tau, \mu) & \approx \mathcal{F}[\rho](\tau, \mu) \\
& =\int \mathrm{e}^{-\mathrm{i} \varphi(t, \boldsymbol{z}, \tau, \mu)} A(\boldsymbol{z}, t, \tau, \mu) \rho(\boldsymbol{z}) d t d \boldsymbol{z}
\end{aligned}
$$

where

$$
\begin{aligned}
& \varphi(t, \boldsymbol{z}, \tau, \mu)=\omega_{0} t\left[\mu-1+\left(\widehat{\gamma_{T}(\tau)}-\mathbf{z} \cdot \dot{\gamma}_{T}(\tau)\right.\right. \\
& \left.\left.+\gamma_{R} \widehat{(\tau)}-\mathbf{z} \cdot \dot{\gamma}_{R}(\tau)\right) / c_{0}\right]
\end{aligned}
$$

and

$$
\begin{aligned}
& A(\boldsymbol{z}, t, \tau, \mu)=\mathrm{e}^{\mathrm{i} \omega_{0}\left(\tau-R_{T R}(\tau, \boldsymbol{z}) / c_{0}\right)} \phi(t) \\
& \times A_{T R}\left(\omega_{0}, \mathbf{z}, \gamma_{R}(t+\tau), \gamma_{T}(t+\tau)\right) \\
& \times \tilde{p}\left[\left(1-\left(\left(\boldsymbol{\gamma}_{T} \widehat{(\tau)}-\mathbf{z} \cdot \dot{\gamma}_{T}(\tau)+\widehat{\gamma_{R}(\tau)}-\mathbf{z} \cdot \dot{\gamma}_{R}(\tau)\right) / c_{0}\right)\right) t\right. \\
& \left.+\tau-R_{T R}(\tau, \mathbf{z}) / c_{0}\right] \tilde{p}^{*}(\mu t) \text {. }
\end{aligned}
$$

We refer to $\mathcal{F}$ as the forward modeling operator.

We use $\omega_{0}=2 \pi f_{0}$ and let $\tilde{f}_{d}=f_{0}(1-\mu)$. The phase of the forward operator becomes

$\varphi(t, \boldsymbol{z}, \tau, \mu)=2 \pi t\left[f_{d}(\tau, \mathbf{z})-f_{0}(1-\mu)\right]=2 \pi t\left(f_{d}(\tau, \mathbf{z})-\tilde{f}_{d}\right)$

where

$$
f_{d}(\tau, \mathbf{z})=f_{0}\left[\gamma_{T} \widehat{(\tau)}-\mathbf{z} \cdot \dot{\gamma}_{T}(\tau)+\gamma_{R} \widehat{(\tau)}-\mathbf{z} \cdot \dot{\gamma}_{R}(\tau)\right] / c_{0} .
$$




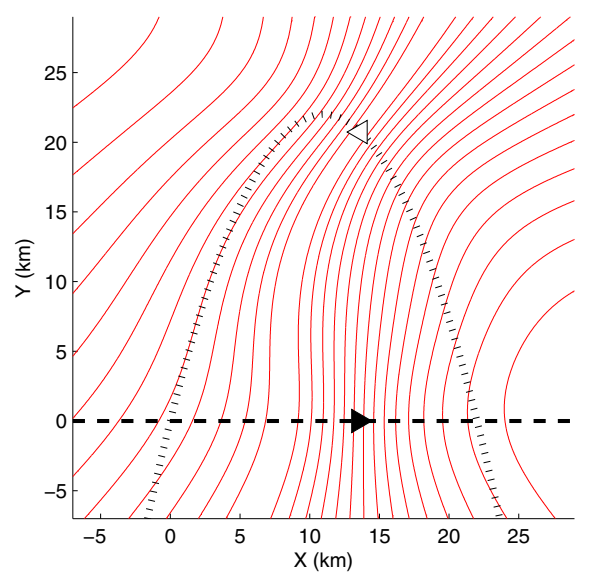

Fig. 1: Iso-Doppler contours $F(\tau, \mu)$ for the Doppler $f_{d}(0.5388 \mathrm{~s}, \mathbf{z})$. The transmitter and receiver are traversing a linear flight trajectory $\gamma_{L}(s)=[s, 0,6.5] \mathrm{km}$ (dashed line) and a parabolic trajectory, $\gamma_{P}(s)=\left[s,\left(22 s-s^{2}\right) 22 / 121,6.5\right] \mathrm{km}$ (dotted line) at the speed of $261 \mathrm{~m} / \mathrm{s}$ over a flat topography, respectively, where black and white triangles denote the positions of the transmitter and receiver at $s=13.5 \mathrm{~km}$ and $s=13.641 \mathrm{~km}$ respectively.

Note that $f_{d}$ is the bistatic Doppler frequency induced by the radial movements of the transmitter and receiver with respect to the scatterer $z$ on the ground.

We assume that for some $m_{A}, A$ satisfies the inequality

$$
\begin{aligned}
\sup _{(t, \mu, \tau, \boldsymbol{z}) \in \mathcal{U}}\left|\partial_{t}^{\alpha_{t}} \partial_{\mu}^{\alpha_{\mu}} \partial_{\tau}^{\beta} \partial_{z_{1}}^{\epsilon_{1}} \partial_{z_{2}}^{\epsilon_{2}} A(\boldsymbol{z}, t, \tau, \mu)\right| \\
\leq C_{A}\left(1+t^{2}\right)^{\left(m_{A}-\left|\alpha_{t}\right|\right) / 2}
\end{aligned}
$$

where $\mathcal{U}$ is any compact subset of $\mathbb{R}^{+} \times \mathbb{R}^{+} \times \mathbb{R} \times \mathbb{R}^{2}$, and the constant $C_{A}$ depends on $\mathcal{U}, \alpha_{t, \mu}, \beta, \epsilon_{1,2}$. This assumption is needed in order to make various stationary phase calculations hold. In practice, (14) is satisfied for radar sufficiently away from the illuminated scene.

Under the assumption (14), (9) defines $\mathcal{F}$ as a Fourier integral operator whose leading-order contribution comes from those points lying in the intersection of the illuminated surface and the constant-Doppler cone $\left\{\mathbf{x} \in \mathbb{R}^{3}: f_{d}(\tau, \mathbf{z})=\right.$ $\left.f_{0}(1-\mu)\right\}$. We denote the curves formed by this intersection by

$$
F(\tau, \mu)=\left\{\boldsymbol{z}: f_{d}(\tau, \mathbf{z})=f_{0}(1-\mu)\right\}
$$

and refer to $F(\tau, \mu)$ as an iso-Doppler contour. For flat topography, $\psi(\boldsymbol{z})=0$, the iso-Doppler contours are given by hyperbolas on the plane $z_{3}=0$. Figure 1 shows the isoDoppler contours for the transmitter traversing a straight line trajectory and the receiver traversing a parabolic trajectory over a flat topography.

\section{IMAGE FORMATION}

\section{A. Filtered Backprojection Operator}

Our objective is to reconstruct $\rho(\boldsymbol{z})$ based on the forward model given by (9). Since $\mathcal{F}$ is a Fourier integral operator, we form the image of $\rho(\boldsymbol{z})$ by a suitable filtered-backprojection of the data $d(\tau, \mu)$ onto $F(\tau, \mu)$ [8].

We form the image by filtered-backprojection as follows:

$$
\begin{aligned}
\tilde{\rho}(\boldsymbol{z}) & :=\mathcal{K}[d](\boldsymbol{z}) \\
& =\int \mathrm{e}^{\mathrm{i} \varphi(t, \boldsymbol{z}, \tau, \mu)} Q(\boldsymbol{z}, t, \tau, \mu) d(\tau, \mu) d t d \tau d \mu
\end{aligned}
$$

where $\mathcal{K}$ is referred to as the filtered-backprojection operator and $Q$ is the filter to be determined below.

\section{B. Point Spread Function of the FBP Operator}

We rewrite $\tilde{\rho}(\boldsymbol{z})$ as

$$
\tilde{\rho}(\boldsymbol{z})=\int L\left(\boldsymbol{z}, \boldsymbol{z}^{\prime}\right) \rho\left(\boldsymbol{z}^{\prime}\right) d \boldsymbol{z}^{\prime}
$$

where $L\left(\boldsymbol{z}, \boldsymbol{z}^{\prime}\right)$ is the point spread function given by

$$
\begin{aligned}
L\left(\boldsymbol{z}, \boldsymbol{z}^{\prime}\right)=\int \mathrm{e}^{\mathrm{i}\left[\varphi(t, \boldsymbol{z}, \tau, \mu)-\varphi\left(t^{\prime}, \boldsymbol{z}^{\prime}, \tau, \mu\right)\right]} & \\
& \times Q(\boldsymbol{z}, t, \tau, \mu) A\left(\boldsymbol{z}^{\prime}, t^{\prime}, \tau, \mu\right) d t d t^{\prime} d \tau d \mu
\end{aligned}
$$

We define

$$
\Phi_{K}:=\varphi(t, \boldsymbol{z}, \tau, \mu)-\varphi\left(t^{\prime}, \boldsymbol{z}^{\prime}, \tau, \mu\right)
$$

and use the stationary phase theorem to approximate the $t^{\prime}$ and $\mu$ integrations of (18). We compute

$$
\partial_{t^{\prime}} \Phi_{K}=f_{d}\left(\tau, \boldsymbol{z}^{\prime}\right)-f_{0}(1-\mu)
$$

and

$$
\partial_{\mu} \Phi_{K}=2 \pi f_{0}\left(t-t^{\prime}\right) .
$$

The stationary points of the phase satisfying $\partial_{t^{\prime}, \mu} \Phi_{K}=0$ imply that

$$
\mu=1-f_{d}\left(\tau, \boldsymbol{z}^{\prime}\right) / f_{0}
$$

and $t=t^{\prime}$.

Substituting the results back into (18), we obtain

$$
\begin{aligned}
L\left(\boldsymbol{z}, \boldsymbol{z}^{\prime}\right)=\int \mathrm{e}^{\mathrm{i} 2 \pi t\left[f_{d}(\tau, \boldsymbol{z})-f_{d}\left(\tau, \boldsymbol{z}^{\prime}\right)\right]} Q\left(\boldsymbol{z}, t, \tau, 1-f_{d}\left(\tau, \boldsymbol{z}^{\prime}\right) / f_{0}\right) \\
\times A\left(\boldsymbol{z}^{\prime}, t, \tau, 1-f_{d}\left(\tau, \boldsymbol{z}^{\prime}\right) / f_{0}\right) d t d \tau .
\end{aligned}
$$

For notational simplicity, we let

$$
\begin{aligned}
Q\left(\boldsymbol{z}, t, \tau, \boldsymbol{z}^{\prime}\right) & =Q\left(\boldsymbol{z}, t, \tau, 1-f_{d}\left(\tau, \boldsymbol{z}^{\prime}\right) / f_{0}\right) \\
A\left(\boldsymbol{z}^{\prime}, t, \tau\right) & =A\left(\boldsymbol{z}^{\prime}, t, \tau, 1-f_{d}\left(\tau, \boldsymbol{z}^{\prime}\right) / f_{0}\right)
\end{aligned}
$$

for the rest of our paper. 


\section{Determination of the FBP Filter}

To determine the filter, we linearize $f_{d}\left(\tau, z^{\prime}\right)$ around $\boldsymbol{z}^{\prime}=\boldsymbol{z}$ using the fundamental theorem of calculus [9] and approximate

$$
f_{d}(\tau, \boldsymbol{z})-f_{d}\left(\tau, \boldsymbol{z}^{\prime}\right) \approx\left(\boldsymbol{z}-\boldsymbol{z}^{\prime}\right) \cdot \boldsymbol{\Xi}\left(\tau, \boldsymbol{z}, \boldsymbol{z}^{\prime}\right)
$$

and

$$
\begin{aligned}
Q\left(\boldsymbol{z}, t, \tau, \boldsymbol{z}^{\prime}\right) & =Q(\boldsymbol{z}, t, \tau, \boldsymbol{z}) \\
A\left(\boldsymbol{z}^{\prime}, t, \tau\right) & =A(\boldsymbol{z}, t, \tau)
\end{aligned}
$$

where in (26)

$$
\boldsymbol{\Xi}\left(\tau, \boldsymbol{z}, \boldsymbol{z}^{\prime}\right)=\int_{0}^{1} \nabla f_{d}\left(\tau, \boldsymbol{z}+\lambda\left(\boldsymbol{z}^{\prime}-\boldsymbol{z}\right)\right) d \lambda .
$$

For notational simplicity, we let $Q(\boldsymbol{z}, t, \tau)=Q(\boldsymbol{z}, t, \tau, \boldsymbol{z})$ for the rest of our paper.

For each $z$, we make the following change of variables:

$$
(t, \tau) \rightarrow \boldsymbol{\xi}=t \boldsymbol{\Xi}(\tau, \boldsymbol{z}, \boldsymbol{z})
$$

in the integral of (23) and then we have

$$
L\left(\boldsymbol{z}, \boldsymbol{z}^{\prime}\right)=\int_{\Omega_{\boldsymbol{z}}} \mathrm{e}^{\mathrm{i} 2 \pi\left(\boldsymbol{z}-\boldsymbol{z}^{\prime}\right) \cdot \boldsymbol{\xi}} Q(\boldsymbol{z}, \boldsymbol{\xi}) A(\boldsymbol{z}, \boldsymbol{\xi}) \eta(\boldsymbol{z}, \boldsymbol{\xi}) d \boldsymbol{\xi}
$$

where

$$
\begin{aligned}
& Q(\boldsymbol{z}, \boldsymbol{\xi})=Q(\boldsymbol{z}, t(\boldsymbol{\xi}), \tau(\boldsymbol{\xi})) \\
& A(\boldsymbol{z}, \boldsymbol{\xi})=A(\boldsymbol{z}, t(\boldsymbol{\xi}), \tau(\boldsymbol{\xi}))
\end{aligned}
$$

and

$$
\eta(\boldsymbol{z}, \boldsymbol{\xi})=\left|\frac{\partial(t, \tau)}{\partial \boldsymbol{\xi}}\right|=\left|\operatorname{det}\left[\begin{array}{c}
\boldsymbol{\Xi}(\tau, \boldsymbol{z}, \boldsymbol{z}) \\
\partial_{\tau} \boldsymbol{\Xi}(\tau, \boldsymbol{z}, \boldsymbol{z})
\end{array}\right]\right|^{-1}
$$

is the determinant of the Jacobian that comes from the change of variables given by (30). The notation det in (33) denotes matrix determinant. In (31),

$$
\Omega_{\boldsymbol{z}}=\left\{\boldsymbol{\xi}=t \boldsymbol{\Xi}(\tau, \boldsymbol{z}, \boldsymbol{z}) \mid A(\boldsymbol{z}, t, \tau) \neq 0,(t, \tau) \in\left(\mathbb{R}^{+}, \mathbb{R}\right)\right\} .
$$

We refer to $\Omega_{z}$ as the data collection manifold at $z$. This set determines many of the properties of the image.

Using (29) and (13), we obtain

$$
\boldsymbol{\Xi}(\tau, \boldsymbol{z}, \boldsymbol{z})=-\frac{f_{0}}{c_{0}} D \psi(\boldsymbol{z}) \cdot\left[\frac{\dot{\boldsymbol{\gamma}}_{T, \perp}(\tau, \boldsymbol{z})}{\left|\gamma_{T}(\tau)-\mathbf{z}\right|}+\frac{\dot{\boldsymbol{\gamma}}_{R, \perp}(\tau, \boldsymbol{z})}{\left|\boldsymbol{\gamma}_{R}(\tau)-\mathbf{z}\right|}\right]
$$

where

$$
D \psi(\boldsymbol{z})=\left[\begin{array}{ccc}
1 & 0 & \partial \psi(\boldsymbol{z}) / \partial z_{1} \\
0 & 1 & \partial \psi(\boldsymbol{z}) / \partial z_{2}
\end{array}\right]
$$

and $\dot{\gamma}_{T(R), \perp}(\tau, \boldsymbol{z})$ is the projection of $\dot{\gamma}_{T(R)}(\tau)$ onto the plane normal to $\widehat{\gamma_{T(R)}(\tau)}-\mathbf{z}$ given by

$$
\begin{aligned}
& \dot{\gamma}_{T(R), \perp}(\tau, \mathbf{z})=\dot{\gamma}_{T(R)}(\tau)- \\
& \gamma_{T(R)}(\tau)-\mathbf{z}\left(\left(\gamma_{T(R)} \widehat{(\tau)}-\mathbf{z}\right) \cdot \dot{\gamma}_{T(R)}(\tau)\right) .
\end{aligned}
$$

We choose the filter as follows:

$$
Q(\boldsymbol{z}, t, \tau)=\chi_{\Omega_{\boldsymbol{z}}}(\boldsymbol{\xi}(t, \tau)) \frac{\overline{A(\boldsymbol{z}, t, \tau)}}{|A(\boldsymbol{z}, t, \tau)|^{2}} \frac{1}{\eta(\boldsymbol{z}, \boldsymbol{\xi})}
$$

where $\chi_{\Omega_{z}}$ is a smooth cut-off function equal to one in the interior of $\Omega_{z}$ and zero in the exterior of $\Omega_{z}$. Note that we choose the filter to make the point spread function $L\left(\boldsymbol{z}, \boldsymbol{z}^{\prime}\right)$ in (31) be the Dirac delta function.

\section{Resolution Analysis}

Substituting (38) into (31) and the result back into (16), we obtain

$$
\begin{aligned}
\tilde{\rho}(\boldsymbol{z}) & :=\mathcal{K} \mathcal{F}[\rho](\boldsymbol{z}) \\
& =\int_{\Omega_{\boldsymbol{z}}} \mathrm{e}^{\mathrm{i} 2 \pi\left(\boldsymbol{z}-\boldsymbol{z}^{\prime}\right) \cdot \boldsymbol{\xi}} \rho\left(\boldsymbol{z}^{\prime}\right) d \boldsymbol{z}^{\prime} d \boldsymbol{\xi} .
\end{aligned}
$$

(39) shows that the image $\tilde{\rho}$ is a band-limited version of $\rho$ whose bandwidth is determined by the data collection manifold $\Omega_{z}$, which describes the many of properties of the image. The larger the data collection manifold, the better the resolution of the reconstructed image is.

Microlocal analysis of (39) shows that an edge at point $\boldsymbol{z}$ is visible if the direction $\boldsymbol{n}_{\boldsymbol{z}}$ normal to the edge is contained in the data collection manifold $\Omega_{z}$ [7], [10]-[12]. Consequently, an edge at point $\boldsymbol{z}$ with $\boldsymbol{n}_{\boldsymbol{z}}$ to the edge is visible if there exists $\tau$ such that $\boldsymbol{\xi}$ is parallel to $\boldsymbol{n}_{\boldsymbol{z}}$. Furthermore, the band-width contribution of $\boldsymbol{\xi}$ to a visible edge is given by

$$
\frac{f_{0}}{c_{0}} L_{\phi}\left|D \psi(\boldsymbol{z}) \cdot\left[\frac{\dot{\boldsymbol{\gamma}}_{T, \perp}(\tau, \boldsymbol{z})}{\left|\boldsymbol{\gamma}_{T}(\tau)-\mathbf{z}\right|}+\frac{\dot{\boldsymbol{\gamma}}_{R, \perp}(\tau, \boldsymbol{z})}{\left|\boldsymbol{\gamma}_{R}(\tau)-\mathbf{z}\right|}\right]\right|
$$

where $L_{\phi}$ denotes the length of the support of $\phi(t)$. Thus, as the frequency of the transmitted signal $f_{0}$ becomes higher, the magnitude of $\boldsymbol{\xi}$ is larger, which contributes to higher image resolution. The longer support of $\phi(t)$ also gives rise to sharper reconstructed edges perpendicular to $\xi$. Additionally, we note that the resolution depends on the range via the terms $\left|\gamma_{T}(\tau)-\mathbf{z}\right|$ and $\left|\gamma_{R}(\tau)-\mathbf{z}\right|$ in (40) and the velocities of the transmitter and receiver via the terms $\dot{\gamma}_{T, \perp}$ and $\dot{\gamma}_{R, \perp}$. As the point is further away from the transmitter and receiver or the velocity of the transmitter, or receiver decreases, the resolution is getting worse due to decreasing of the magnitude of $\boldsymbol{\xi}$.

(39) shows that irrespective of the choice of the filter, the backprojection operator recovers the visible edges of the scene at the correct location. With the choice of filter given in (38), the resulting image formation algorithm can recover not only the correct location and orientation, but also the correct strength of the visible edges [10], [12]-[14].

\section{Numerical Simulations}

We performed numerical simulations for a point target, i.e., reconstructed the point spread function (PSF) numerically under different scenarios to demonstrate the theoretical results described in section III.

We considered a scene of size $[0,1100] \times[0,1100] \mathrm{m}^{2}$ with flat topography centered at $[11,11,0] \mathrm{km}$. The scene was discretized by $128 \times 128$ pixels, where $[0,0,0] \mathrm{m}$ and $[1100,1100,0] \mathrm{m}$ corresponds to the pixels $(1,1)$ and $(128,128)$, respectively.

The point target was assumed to be at $[550,954,0] \mathrm{m}$ in the scene considered. This position corresponds to the $(65,112)$ th pixel in the reconstructed scene. 


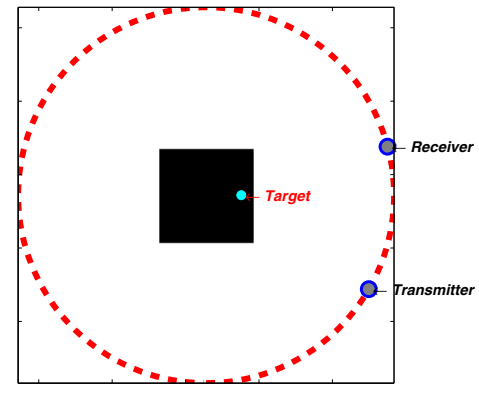

Fig. 2: The 2-D view of the simulation setup. The dark region denotes the scene considered in the simulations. The light blue solid circle indicates the location of the point target. The circular trajectory is shown by the red dashed line. At a certain time instant, the transmitter and the receiver are located at the positions shown in the figure.

We assumed that the transmitter and receiver were traversing the same circular trajectory given by

$$
\gamma_{C}(s)=(11+22 \cos (s), 11+22 \sin (s), 6.5) \mathrm{km} .
$$

Let $\gamma_{T}(s)$ and $\gamma_{R}(s)$ denote the trajectories of the two receivers. We set $\gamma_{T}(s)=\gamma_{C}(s)$ and $\gamma_{R}(s)=\gamma_{1}\left(s-\frac{\pi}{4}\right)$. Note that the variable $s$ in $\gamma_{C}$ is equal to $\frac{V}{R} t$ where $V$ is the speed of the receiver, and $R$ is the radius of the circular trajectory. We set the speed of the two receivers to $261 \mathrm{~m} / \mathrm{s}$.

Fig. 2 shows $2 \mathrm{D}$ view of the scene with the target, transmitter and receiver trajectories.

For all the experiments, we assumed that the transmitter transmited a single-frequency continuous waveform, i.e., $\tilde{p}(t)$ in 2 was set to be unit. We used 6 to generate the data and chose the windowing function $\phi$ in 7 to be a Hanning function.

We performed simulations to demonstrate the impact of $L_{\phi}$, the length of the support of the $\phi(t) ; f_{0}$, the carrier frequency of the transmitted waveform and $f_{\tau}$, the sampling rate of $\tau$ on the PSF of the imaging operator. The PSF were reconstructed with the following variables: (1) $f_{0}=$ $800 \mathrm{MHz}, L_{\phi}=0.0107 \mathrm{~s}, f_{\tau}=0.4834 \mathrm{~Hz}$; 2) $f_{0}=800 \mathrm{MHz}$, $L_{\phi}=0.3413 \mathrm{~s}, f_{\tau}=0.4834 \mathrm{~Hz}$; (3) $f_{0}=80 \mathrm{MHz}, L_{\phi}=$ $0.3413 \mathrm{~s}, f_{\tau}=0.4834 \mathrm{~Hz}$; (4) $f_{0}=800 \mathrm{MHz}, L_{\phi}=0.0107 \mathrm{~s}$, $f_{\tau}=96.6736 \mathrm{~Hz}$. Note that $f_{\tau}=0.4834 \mathrm{~Hz}$ and $f_{\tau}=$ $96.6736 \mathrm{~Hz}$ correspond to the uniform sampling of the circular trajectory with 256 and 51200 points, respectively.

Fig. 3-Fig. 6 show the reconstructed PSFs along with their profiles in $\mathrm{X}$ and $\mathrm{Y}$ directions for the four cases.

As can be seen in Fig. 3 and Fig. 4, the quality of the PSF improves as the length of the support of the windowing function increases from $0.0101 \mathrm{~s}$ to $0.3413 \mathrm{~s}$ because of a larger data collection manifold. We see that the level of the sidelobes of the PSF decreases as the support of windowing function gets longer, which results in an increase in the image contrast. Recalling that our imaging method relies on the Doppler ambiguity of the transmitted waveform, the results are

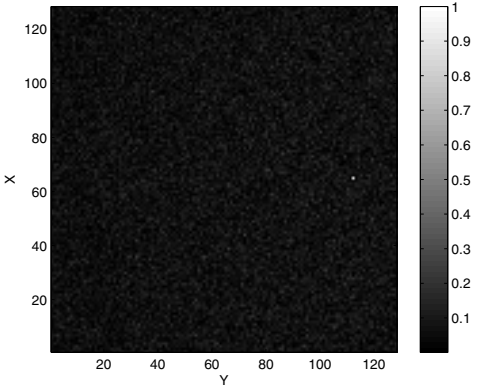

(a)

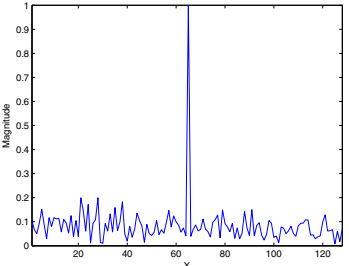

(b)

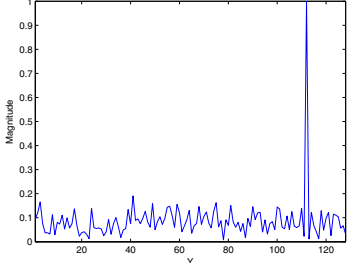

(c)
Fig. 3: (a) The reconstructed PSF at $[550,954,0] \mathrm{m}$ along with (b) its X profile and (c) Y profile, for a transmitter transmitting a single-frequency $\mathrm{CW}$ waveform at $f_{0}=800 \mathrm{MHz}$ with $L_{\phi}=$ $0.0107 \mathrm{~s}$ and $f_{\tau}=0.4834 \mathrm{~Hz}$.

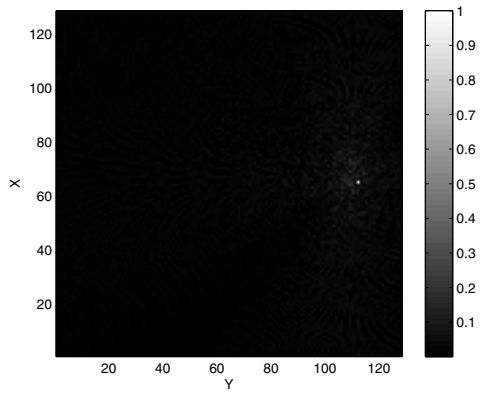

(a)

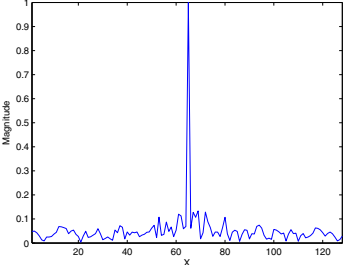

(b)

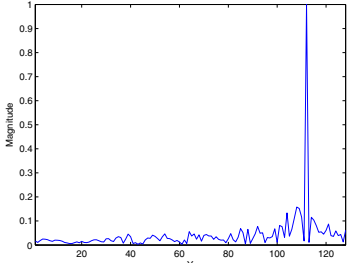

(c)
Fig. 4: (a) The reconstructed PSF at $[550,954,0] \mathrm{m}$ along with (b) its X profile and (c) Y profile, for a transmitter transmitting a single-frequency $\mathrm{CW}$ waveform at $f_{0}=800 \mathrm{MHz}$ with $L_{\phi}=$ $0.3413 \mathrm{~s}$ and $f_{\tau}=0.4834 \mathrm{~Hz}$.

consistent with the theory that the Doppler ambiguity improves as the length of signal in time domain increases. 


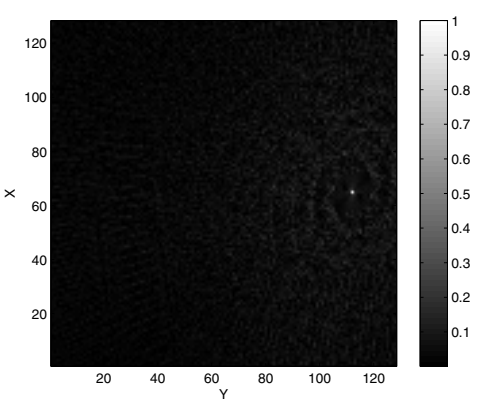

(a)

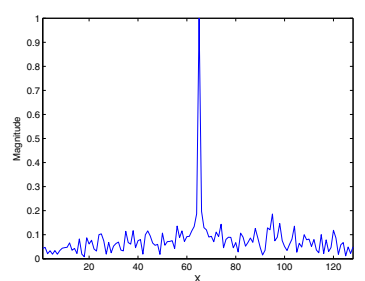

(b)

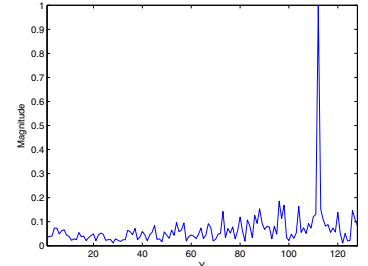

(c)
Fig. 5: (a) The reconstructed PSF at $[550,954,0] \mathrm{m}$ along with (b) its X profile and (c) Y profile, for a transmitter transmitting a single-frequency $\mathrm{CW}$ waveform at $f_{0}=80 \mathrm{MHz}$ with $L_{\phi}=$ $0.3413 \mathrm{~s}$ and $f_{\tau}=0.4834 \mathrm{~Hz}$.

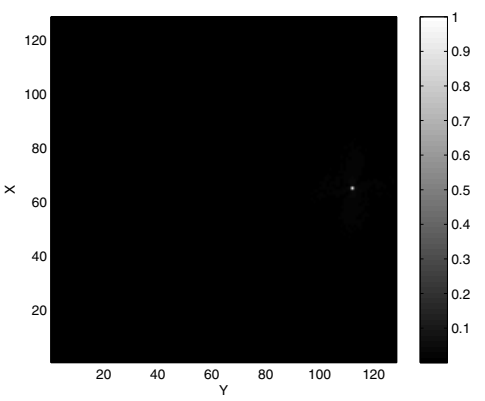

(a)

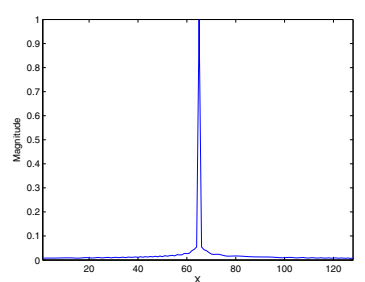

(b)

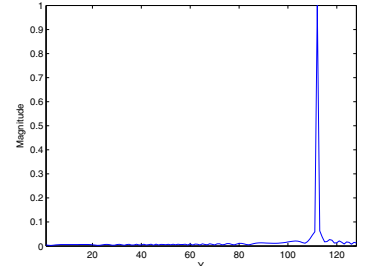

(c)
Fig. 6: (a) The reconstructed PSF at $[550,954,0] \mathrm{m}$ along with (a) its X profile and (c) Y profile, for a transmitter transmitting a single-frequency $\mathrm{CW}$ waveform at $f_{0}=800 \mathrm{MHz}$ with $L_{\phi}=$ $0.0107 \mathrm{~s}$ and $f_{\tau}=96.6736 \mathrm{~Hz}$.

Comparing Fig. 5 with Fig. 4, we see that the quality of the PSF degrades as the carrier frequency of the transmitted waveform decreases from $800 \mathrm{MHz}$ to $80 \mathrm{MHz}$ as indicated by the theory. We observed the increase of the level of the sidelobes and the spread at the bottom of the mainlobe in Fig. 5(b) and Fig. 5(c).

Observing Fig. 6 and Fig. 3, we can see that the the increase of the sampling rate of $\tau$, which implies much more samples of $\tau$ also leads to a clear improvement of the reconstructed PSF. There is a significant improvement in the suppression of the sidelobes of the PSF in Fig. 6(b) and Fig. 6(c).

\section{CONCLUSION}

In this paper, we developed a novel image formation method for SAR that uses ultranarrow-band transmitted waveforms where the transmitter and receiver may traverse arbitrary trajectories. This method is based on the windowed, scaled and translated correlation of the received signal and transmitted waveform and a filtered backprojection of the resulting correlated signal. The numerical simulations demonstrate the performance of the reconstruction.

\section{REFERENCES}

[1] D. Mensa, S. Halevy, and G. Wade, "Coherent doppler tomography for microwave imaging," Proceedings of the IEEE, vol. 71, pp. 254-261, Feb. 1983.

[2] D. Mensa and G. Heidbreder, "Bistatic synthetic-aperture radar imaging of rotating objects," IEEE Transactions on Aerospace and Electronic Systems, vol. 18, pp. 423-431, July 1982.

[3] H. Sun, H. Feng, and Y. Lu, "High resolution radar tomographic imaging using single-tone cw signals," in 2010 IEEE Radar Conference, May 2010, pp. 975-980.

[4] M. C. Wicks, B. Himed, J. L. E. Bracken, H. Bascom, and J. Clancy, "Ultra narrow band adaptive tomographic radar," in IEEE International workshop on computational advances in multi-sensor adaptive processing, Dec. 2005, pp. 36-39.

[5] S. L. Coetzee, C. J. Baker, and H. D. Griffiths, "Narrow band high resolution radar imaging," in 2006 IEEE Radar Conference, April 2006, pp. 622-625.

[6] B. Borden and M. Cheney, "Synthetic-aperture imaging from highdoppler-resolution measurements," Inverse Problems, vol. 21, pp. 1-11, Nov. 2005.

[7] C.J. Nolan and M. Cheney, "Synthetic aperture inversion for arbitrary flight paths and non-flat topography," IEEE Transactions on Image Processing, vol. 12, pp. 1035-1043, 2003.

[8] F. Treves, Introduction to Pseudodifferential and Fourier Integral Operators, volumes I and II, Plenum Press, New York, 1980.

[9] R. Courant and F. John, Introduction to Calculus and analysis, vol. 1 and 2, Springer, New York, 2000.

[10] C. J. Nolan and M. Cheney, "Synthetic aperture inversion," Inverse Problems, vol. 18, pp. 221-236, 2002.

[11] E.T. Quinto, "Singularities of the x-ray transform and limited data tomography in $r^{2}$ and $r^{3}$," SIAM J. Math. Anal., vol. 24, pp. 1215-1225, 1993.

[12] B. Yazici, M. Cheney, and C.E. Yarman, "Synthetic-aperture inversion in the presence of noise and clutter," Inverse Problems, vol. 22, pp. 1705-1729, 2006

[13] C.J. Nolan and M. Cheney, "Microlocal analysis of synthetic aperture radar imaging," The Journal of Fourier Analysis and Applications, vol. 10, pp. 133-148, 2004

[14] C.E. Yarman, B. Yazıc1, and M. Cheney, "Bistatic synthetic aperture radar imaging for arbitrary flight trajectories," Image Processing, IEEE Transactions on, vol. 17, no. 1, pp. 84-93, Jan. 2008. 